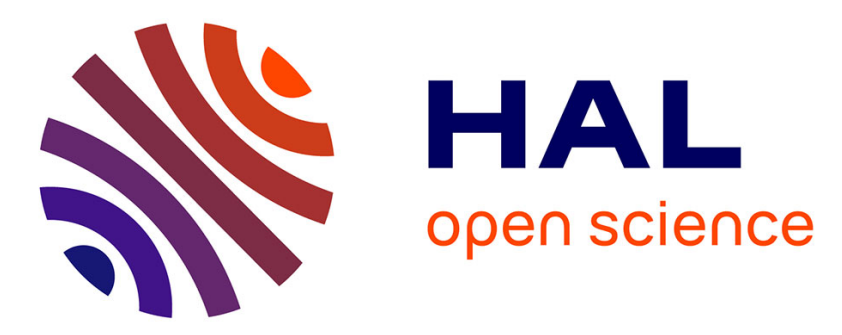

\title{
Dielectric relaxation induced by a space charge in poled glasses for nonlinear optics
}

Marc Dussauze, Olivier Bidault, Evelyne Fargin, Mario Maglione, Vincent Rodriguez

\section{- To cite this version:}

Marc Dussauze, Olivier Bidault, Evelyne Fargin, Mario Maglione, Vincent Rodriguez. Dielectric relaxation induced by a space charge in poled glasses for nonlinear optics. Journal of Applied Physics, 2006, 100 (3), pp.034905/1-7. 10.1063/1.2218998 . hal-00109399

\section{HAL Id: hal-00109399 \\ https://hal.science/hal-00109399}

Submitted on 29 Apr 2021

HAL is a multi-disciplinary open access archive for the deposit and dissemination of scientific research documents, whether they are published or not. The documents may come from teaching and research institutions in France or abroad, or from public or private research centers.
L'archive ouverte pluridisciplinaire HAL, est destinée au dépôt et à la diffusion de documents scientifiques de niveau recherche, publiés ou non, émanant des établissements d'enseignement et de recherche français ou étrangers, des laboratoires publics ou privés. 


\title{
Dielectric relaxation induced by a space charge in poled glasses for nonlinear optics
}

\author{
M. Dussauze, O. Bidault, ${ }^{\text {a) }}$ E. Fargin, and M. Maglione \\ ICMCB, UPR 9048 CNRS, Université Bordeaux 1, 87 Avenue Dr. Schweitzer, 33608 Pessac Cedex, France \\ V. Rodriguez \\ LPCM, UMR 5803 CNRS, Université Bordeaux 1, 351 cours de la Libération, 33405 Talence Cedex, \\ France
}

(Received 8 November 2005; accepted 23 May 2006; published online 2 August 2006)

\begin{abstract}
Unpoled and efficient nonlinear optical (NLO) poled sodium niobium borophosphate glasses have been studied by means of dielectric spectroscopy. The thermal poling process induces a dielectric relaxation that gradually vanishes on heating for $T \gtrsim 500 \mathrm{~K}$. The data are interpreted by a two-layer (Maxwell-Wagner) model, consisting of a bulk and a $\mathrm{Na}^{+}$poor surface layer with lower conductivity. Our results suggest that the ion transport is due to hopping mechanism implying at least $\mathrm{Na}^{+}$. All the dielectric results are in full agreement with previous optical analyses. This is an example of poled glasses where an efficient NLO response is directly correlated to the conductivity of the material enhanced by a high alkali loading. () 2006 American Institute of Physics. [DOI: $10.1063 / 1.2218998]$
\end{abstract}

\section{INTRODUCTION}

In the field of optical communications, glasses with nonlinear optical properties are widely investigated with the aim to supplant ferroelectric crystalline materials for applications, such as integrated electro-optic modulators or frequency converters. As a matter of fact, several recent publications have evidenced consequent $\chi^{(2)}$ responses in lead silicate glasses, ${ }^{1}$ chalchogenide glasses, ${ }^{2}$ and niobium borophosphate glasses ${ }^{3}$ after a thermal poling treatment. The symmetry breaking of isotropic glasses is obtained by applying a high $\mathrm{dc}$ voltage at an elevated temperature $\left(\sim 200-400{ }^{\circ} \mathrm{C}\right)$ and then by cooling the sample down to room temperature keeping the dc field on. A lot of investigations have been reported on the physical mechanism that drives the second-order nonlinearities in poled glasses, especially in silica. In the early 1990s, Myers et al. ${ }^{4}$ have proposed a model where the $\chi^{(2)}$ response induced by the thermal poling process may be expressed as a sum of two terms,

$$
\chi^{(2)} \propto \Sigma \frac{N p \beta}{5 k T} E_{\mathrm{loc}}+3 \chi^{(3)} E_{\mathrm{int}} .
$$

The first term results from the possible reorientation of polar bonds, each of them having a permanent dipole $p(N$ is the density number of permanent dipoles) and a hyperpolarizability $\beta$. The second term is related to the interaction between the third-order nonlinearity $\chi^{(3)}$ and the residual internal electric field $E_{\text {int }}$ induced by a space charge migration of mobile ions at the anode side, expanding typically over a few micrometers. ${ }^{5,6}$ To date, no clear evidence of the contribution of the first term has been yet established in pure glass materials. Therefore, synthesis engineering of glass materials dedicated to nonlinear optical applications has focalized on

\footnotetext{
${ }^{a)}$ Author to whom correspondence should be addressed; electronic mail: obidault@u-bourgogne.fr
}

compositions that increase the third-order nonlinearity response. ${ }^{7}$ Noticeably, all $\chi^{(2)}$ designed glass compositions that one may find in the literature are poor ionic conductive materials where the mobile charge species stand more at the level of impurities, i.e., a few ppm of $\mathrm{Na}^{+}, \mathrm{Li}^{+}, \mathrm{OH}^{-}$, etc., like in silica ${ }^{9}$ or $20 \mathrm{WO}_{3}-80 \mathrm{TeO}_{2}$ (Ref. 10) glasses.

Among glass materials, the borophosphate matrix is indeed very suitable for nonlinear optical (NLO) applications. ${ }^{11}$ Our engineering composition purpose was first that the introduction of $\mathrm{Nb}-\mathrm{O}$ entities would increase the polarizability of the whole material and second that the adjunction of mobile ionic species, $\mathrm{Na}^{+}$, at a high level of concentration would improve the space charge migration during the poling process. Recently, several results obtained on sodium and niobium borophosphate poled bulk glasses with compositions $(1-x)\left(0.95 \mathrm{NaPO}_{3}+0.05 \mathrm{Na}_{2} \mathrm{~B}_{4} \mathrm{O}_{7}\right)+x \mathrm{Nb}_{2} \mathrm{O}_{5}$, for $x \leqslant 0.5$, have been reported. ${ }^{12}$ Notably, we were reproducibly able to induce homogeneous $\chi^{(2)}$ signals, as high as $5 \mathrm{pm} / \mathrm{V}$, on large area of $\sim 0.5 \mathrm{~cm}^{2}$ in all poled bulk glass plates. First, from this previous work, the second harmonic generation (SHG) efficiency has been correlated to the existence of local three-dimensional niobium oxygen frameworks within the material that amplify the $\chi^{(3)}$ response. Second, a depletion of sodium concentration in a thin layer of $\sim 5 \mu \mathrm{m}$ has been clearly observed at the anode side of the poled plates. A depth profile ( $z$ direction) of the internal static electric field could be deduced and it pointed out a very good agreement with the $z$ profile of simulated $\chi^{(2)}$, obtained from an original analysis of both transmitted and reflected polarized Maker fringe patterns. ${ }^{3}$

We report in this paper impedance spectroscopy characterization of unpoled, poled, and thermally depoled glasses with the composition $0.57\left(0.95 \mathrm{NaPO}_{3}+0.05 \mathrm{Na}_{2} \mathrm{~B}_{4} \mathrm{O}_{7}\right)$ $+0.43 \mathrm{Nb}_{2} \mathrm{O}_{5}$. The purpose of this study is to investigate how 
the macroscopic dielectric response can give more insight into the mechanism of thermal poling treatment in these glasses.

\section{MATERIALS AND EXPERIMENTS}

Bulk samples with composition $0.57\left(0.95 \mathrm{NaPO}_{3}\right.$ $\left.+0.05 \mathrm{Na}_{2} \mathrm{~B}_{4} \mathrm{O}_{7}\right)+0.43 \mathrm{Nb}_{2} \mathrm{O}_{5}$ were produced by a classical solid phase process. High purity reagent powders of $\mathrm{NaPO}_{3}$, $\mathrm{Na}_{2} \mathrm{~B}_{4} \mathrm{O}_{7}$, and $\mathrm{Nb}_{2} \mathrm{O}_{5}$ were mixed and grounded, poured in a platinum crucible and melted at a temperature of $1300{ }^{\circ} \mathrm{C}$. The melt was quenched in a brass preform (1 $\mathrm{mm}$ depth). The glass formed was annealed under air, $10^{\circ} \mathrm{C}$ under the glass transition temperature. Transparent 700-800 $\mu \mathrm{m}$ thickness plates were cut and polished on both sides.

Thermal poling was performed under air at normal pressure. The glass plates were heated at the poling temperature for at least $1 \mathrm{~h}$ before applying the dc electric field during $30 \mathrm{~min}$. Sample plates were subsequently cooled down to room temperature before removing the dc bias. At the anode, low ionic conduction materials (silicon, stainless steal) were used to avoid charge injection into the sample during the poling process. At the cathode, a soda lime glass has been used as a nonblocking electrode to prevent locally the reduction of the sample. Contacts between each electrode and the sample have been carefully checked in order to preserve a good optical quality after the thermal poling treatment. Thermal poling conditions were optimized: each sample was poled at $230{ }^{\circ} \mathrm{C}$ under a $2 \mathrm{kV}$ voltage during $30 \mathrm{~min}$. Under this experimental condition, the applied poling field is close to the material dielectric breakdown as reported in a previous work. ${ }^{3}$ Altogether, 3 unpoled plates and 15 poled plates have been used to check the reproducibility of the measurements; the only parameter that was allowed to change was the sample dimension. Only the results concerning four of them will be presented here.

Prior to the measurements, the faces of the $\sim 700 \mu \mathrm{m}$ plates were electroded with platinum using rf magnetron sputtering. Gold or silver was avoided because a diffusion takes place on heating the sample above $200{ }^{\circ} \mathrm{C}$. The dielectric response was measured under a weak ac field $(\sim 0.1 \mathrm{kV} / \mathrm{cm})$ using an HP 4194 impedance analyzer covering a frequency range from $100 \mathrm{~Hz}$ to $10 \mathrm{MHz}$. The temperature was controlled using a chromel-alumel thermocouple directly in thermal contact with the sample; it could be monitored between 300 and $700 \mathrm{~K}$ with an accuracy better than $0.5 \mathrm{~K}$. The impedance measurements were performed using a dynamic method under a linear temperature variation (typically $\pm 1.5 \mathrm{~K} / \mathrm{min}$ ) in order to record the permittivity $\varepsilon$, the conductivity $\sigma$, or the impedance $Z$ as a function of the temperature at several spot frequencies. The conductivity spectra $\sigma(\omega)$ were recorded on heating in the same conditions. A full spectral acquisition lasts only about $5 \mathrm{~s}$ so that the temperature change is less than $0.1 \mathrm{~K}$. This procedure enables us to obtain the whole data between 300 and $650 \mathrm{~K}$ for $4 \mathrm{~h}$. Each set of experiments has been done on a new fresh poled glass.

As the samples have been poled at $500 \mathrm{~K}$, the thermally activated depoling of poled plates was expected to take place around this temperature range. The depoling process has been experimentally evidenced by recording, without any bias field, a dc current as a function of time at a fixed temperature, using a Keithley 236 electrometer. While for temperatures lower than $500 \mathrm{~K}$ this process could be safely ignored, under our experimental recording conditions we have estimated that it would affect our dielectric measurements for $T \gtrsim 600 \mathrm{~K}$. For this reason, each set of experiments has to be done quickly on a new fresh poled plate and stopped at $650 \mathrm{~K}$, temperature at which the depoling kinetic becomes comparable with the measurement time.

\section{RESULTS AND DISCUSSION}

\section{A. Conductivity curves of unpoled samples}

Let us first consider the conductivity curves of samples before any poling treatment, simultaneously as a function of temperature and frequency. Clearly, the observed conductivity spectra reported in Fig. 1 are typical of a glass. ${ }^{13}$ At low frequencies, the conductivity is independent of the frequency and corresponds to the dc conductivity $\left(\sigma_{\mathrm{dc}}\right)$ whereas one notes a dispersion at higher frequencies. Within this standard frequency window and this temperature range, the frequency response of the (real part of the) conductivity is currently described by

$$
\sigma(\omega)=\sigma_{\mathrm{dc}}+A \omega^{n} .
$$

It decomposes as the sum of a constant de conductivity and a fractional frequency power-law dependence $(n<1)$ with a prefactor $A$. When increasing the temperature, the frequency at which the conductivity deviates from the constant $\sigma_{\mathrm{dc}}$ value is shifted to a higher value [Fig. 1(b)], implying in our restricted frequency range $(100 \mathrm{~Hz}-1 \mathrm{MHz})$ a gradual decrease of the dispersion [Fig. 1(a)]. All the spectra could be fitted using this simple model [i.e., Eq. (2)]. Therefore, it is found that the power-law regime is much less temperature dependent than the dc conductivity. In a first approximation, the exponent $n$ may be considered as constant, $n \approx 0.65$, which is a very common value for oxide glasses. ${ }^{14}$ In the same time, $\sigma_{\mathrm{dc}}$ glass shows an Arrhenius behavior with an activation energy of $0.72 \mathrm{eV}$ (see also Fig. 7).

\section{B. Study of the poling/depoling processes}

A first qualitative insight into the electrical properties of poled samples may be obtained when plotting the $\sigma(T)$ curves of the first heating and cooling runs, at three different frequencies (Fig. 2). Unlike to the case of unpoled plates, a strong frequency dispersion takes place on heating for $T$ $\gtrsim 500 \mathrm{~K}$, revealing a new process. Moreover a thermal hysteresis, not evidenced in the raw glass, appears in the high temperature region. One may note that upon a frequency increase, this thermal hysteresis shifts toward higher temperature $(500 \mathrm{~K}$ at $3 \mathrm{kHz}$ and 580 at $30 \mathrm{kHz})$. After a first thermal heating and cooling run (provided a sufficiently high enough temperature has been reached), the subsequent frequency dependent electrical behavior of the glass has been checked to be identical to the unpoled one, and, in particular, the thermal hysteresis and the high temperature dispersion have disappeared. In parallel, we have observed the disap- 

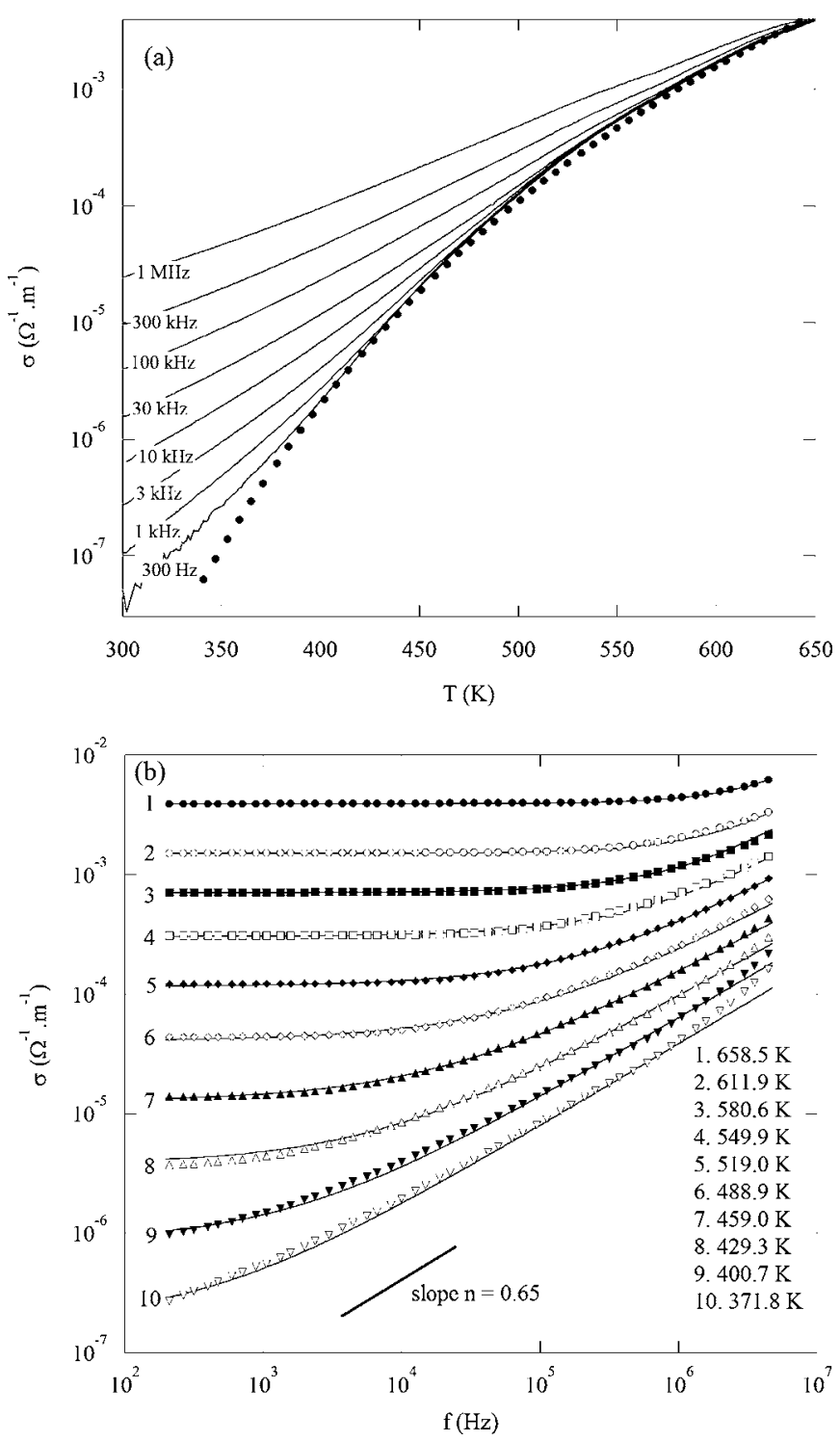

FIG. 1. Conductivity curves of an unpoled sodium borophosphate glass plate as a function of $T$, at some frequencies (a) and as a function of the frequency for some fixed temperatures (b). The $\sigma_{\mathrm{dc}}$ values $(\mathbf{O})$ deduced from the $\sigma(\omega)$ fits according to Eq. (2) are reported in (a). The reported measurements were performed on heating and they do not differ from those obtained on cooling.

pearance of the SHG signal after such a thermal run. These experimental observations tend to show the occurrence of some reversible state between poled and thermally depoled samples.

According to Godbout and Lacroix ${ }^{15}$ the thermal reversibility of nonlinear optical properties are due to the thermal diffusion of sodium ions that shrinks progressively the extent of the nonlinear depleted layer until disappearance. In agreement with this scheme, the depoling process of our glasses would be characterized by a decrease of the dc current as a function of time, without applying any bias field at an isotherm. To get information about the kinetics of this process, the depoling current has been recorded as a function of time during isotherm in the hysteretic temperature range. As an illustration, Fig. 3 reports such a net decrease of the dc current with time at $620 \mathrm{~K}$. In addition, one may note that the decrease is far from a monoexponential behavior.

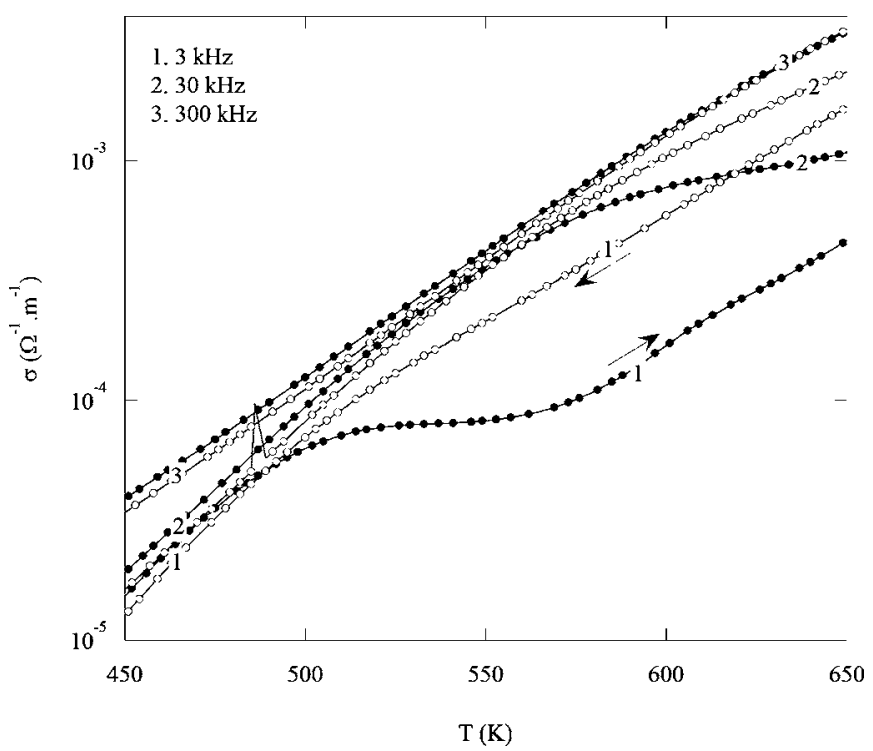

FIG. 2. Conductivity of the poled sodium borophosphate glass as a function of $T$ at different selected frequencies $(3,30$, and $300 \mathrm{kHz})$. The measurements were performed during the first thermal cycle [heating $(\bullet)+$ cooling (O) at a rate of $\pm 1.5 \mathrm{~K} / \mathrm{min}$.

The most common phenomenological functions used for describing the time dependence of decreasing processes are the biexponential, the stretched exponential, the inverse logarithmic, or the power laws. Except the biexponential function, all other single functions are the sum of a constant term $C$ plus a scaled time dependent term $B f(t)$ extrapolating towards zero as time goes to infinity. The coefficient $C(=0$ in the present case) is the asymptotic value while the coefficient $B$ is the magnitude of the time dependent part. Moreover, because the beginning of the experiments is not perfectly defined, a delay $t_{0}$ can be added as an adjustable parameter. It is not easy to discriminate between these functions the one that describes the experimental data, especially because of the limited duration of the experiment. Furthermore, the irreversibility of the change implies that a new sample should

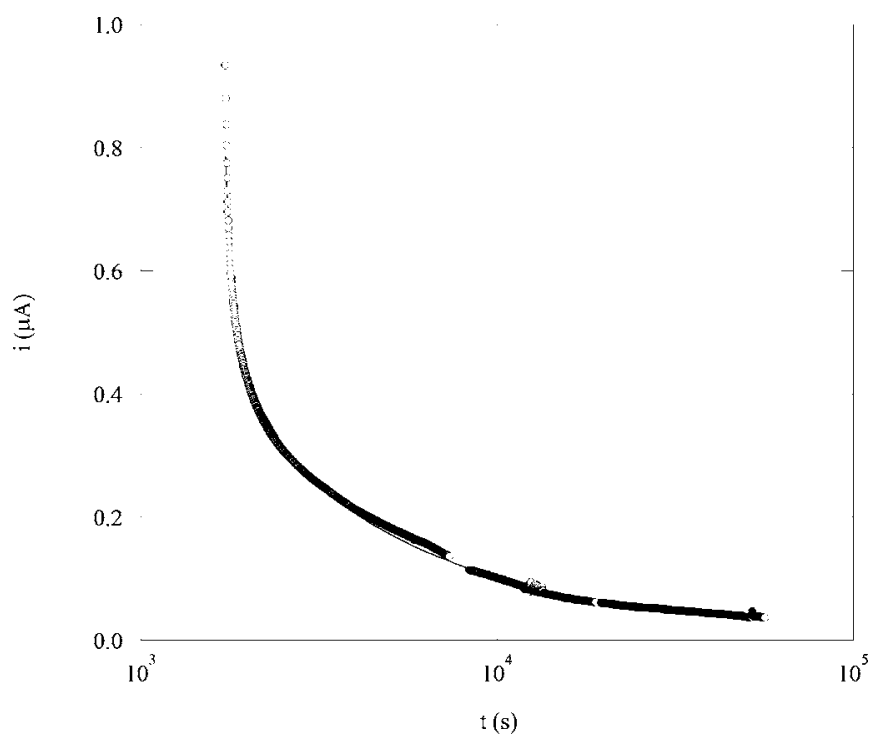

FIG. 3. Experimental depoling current (points) at a fixed temperature $(620 \mathrm{~K})$ as a function of time together with the best fit to Eq. (3) (solid line). 
be used for each isotherm. However, in glass materials, the stretched exponential function is known to be one of the more convenient one that describes a wide distribution of relaxation mechanisms. For a decreasing dc current, it reads

$$
i(t)=C+B \exp \left[-\left(\frac{t-t_{0}}{\tau^{*}}\right)^{\gamma}\right],
$$

where $\tau^{*}$ is the dc current relaxation time, $B$ is the magnitude of the dc current decreasing with time, and $\gamma$ is the exponential power for which a departure from 1 (corresponding to a pure monoexponential behavior) indicates a broadening of the relaxation time distribution, i.e., the stretched exponential function might be substituted by an infinity of monoexponential functions.

Qualitative trends of the experimental depoling curves that can be drawn upon increasing temperature are, first, an increase of both the relaxation rate $\left(1 / \tau^{*}\right)$ and the amplitude factor $B$ and, second, a net departure from a simple monoexponential behavior. For example, the best fits to the experimental curves according to Eq. (3) gave $\tau^{*}=9300 \mathrm{~s}$ and $\gamma$ $=0.6$ at $570 \mathrm{~K}$, and $\tau^{*}=300 \mathrm{~s}$ and $\gamma=0.3$ at $620 \mathrm{~K}$. Indeed, the results obtained at $570 \mathrm{~K}$, slightly above the poling temperature $(\sim 500 \mathrm{~K})$, seem to be consistent since the exponential power value $\gamma=0.6$ ranges typical values of complex relaxation mechanisms in oxide glasses. ${ }^{16}$ In opposition, the unusually lower value obtained at $620 \mathrm{~K}, \gamma=0.3$, far above the poling temperature, might be considered with great care. This strong departure from $\sim 0.6$ might be attributed to an inhomogeneous sample that could be described as a kinetic dependent mixture of poled and depoled areas. Nevertheless, as depicted at the end of Sec. II, the dielectric relaxation measurements of poled samples could be extended up to $T$ $<650 \mathrm{~K}$ since the kinetic dependence of the thermally activated depoling process is partially quenched by the quickness of the dielectric measurements.

In this extended temperature range $(T<650 \mathrm{~K})$, we have thus shown that first poled glasses spectrally behave in a different manner than unpoled or depoled samples and second a dispersive (and reversible) process occurs for $T$ $>500 \mathrm{~K}$ (for $1 \mathrm{kHz}$ ) during the first heating run of poled samples.

\section{Evidence of a monolayer structure in unpoled glasses versus a bilayer structure in poled glasses}

Figure 4 gathers the impedance complex plane (ColeCole) plots obtained at two selected temperatures (600 and $630 \mathrm{~K}$ ) during two distinct heating runs of a poled plate and an unpoled plate, respectively.

For an ideal homogeneous dielectric material, its resistance $R$ and its capacitance $C$ can be computed from a single parallel $R C$ equivalent circuit. The impedance complex plane $\left(Z^{\prime \prime}, Z^{\prime}\right)$ plot (Cole-Cole plot) of this $R C$ element gives rise to one semicircular arc which intercepts the $Z^{\prime}$ axis at zero $(\omega \rightarrow \infty)$ and $R(\omega \rightarrow 0)$. $C$ can be then calculated from the relationship $\omega_{\max } R C=1$, where $\omega_{\max }=2 \pi f_{\max }$ and the top of the semicircular arc determines the frequency $f_{\max }$. The conductivity $\sigma$ and the dielectric permittivity $\varepsilon$ can be then calculated from $R$ and $C$, respectively.
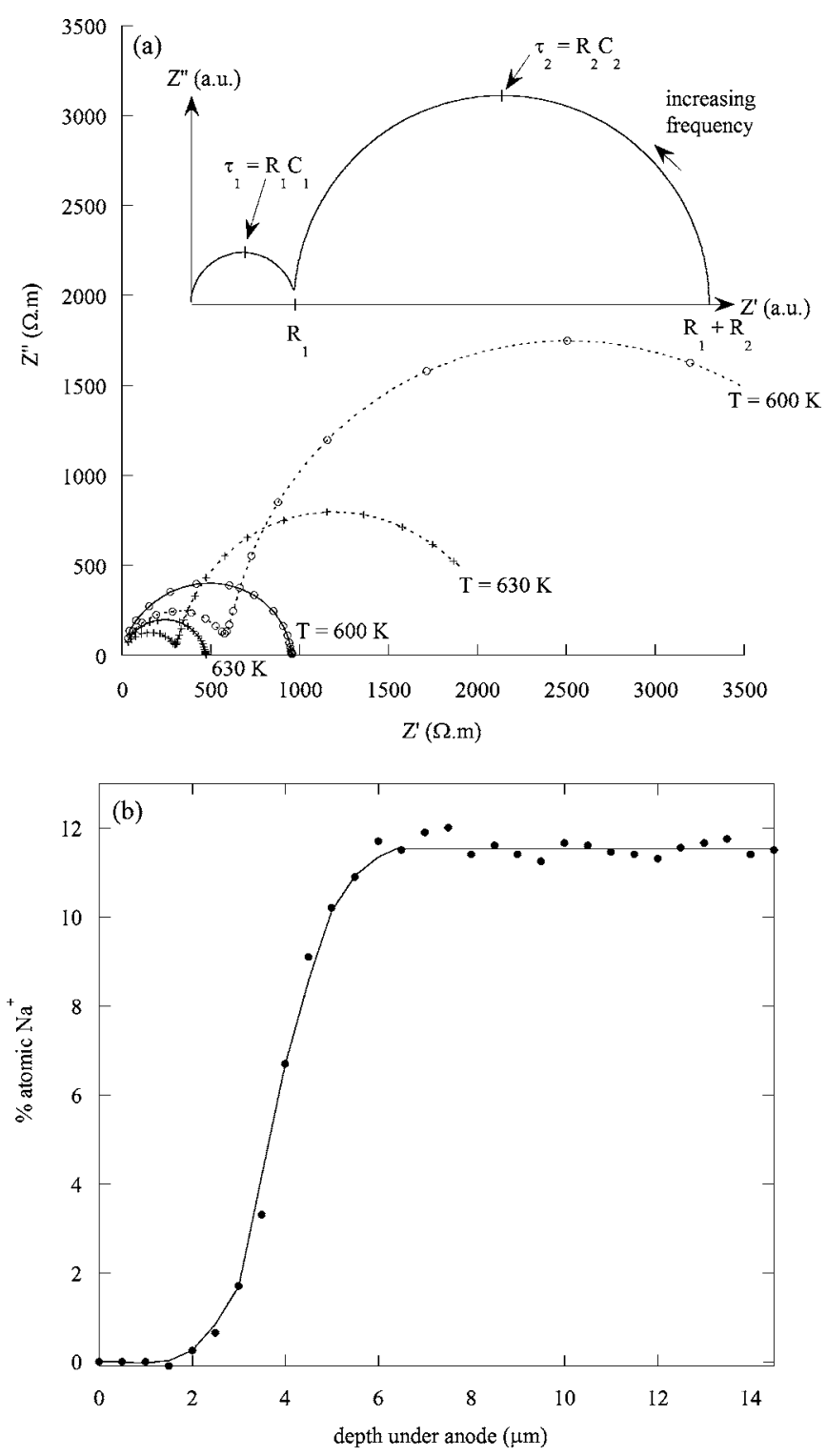

FIG. 4. Upper part: complex impedance plane plot at $600 \mathrm{~K}(\bigcirc)$ and $630 \mathrm{~K}$ $(+)$ for an unpoled (solid line) and poled (dashed line) plate together with the schematic response of an ideal two-layer sample (lines). The impedances are expressed in $\Omega \mathrm{m}$ instead of $\Omega$ in order to allow comparison between the two samples. Lower part: energy dispersive x-ray spectroscopy (EDS) depth profiles of the sodium concentration from $0 \mu \mathrm{m}$ (anode surface) to $15 \mu \mathrm{m}$ inside the glass (from Ref. 3).

The unpoled glass Cole-Cole plots exhibit a quite perfect semicircle, although a deviation from this ideal behavior occurs at high frequencies $\left(Z^{\prime} \rightarrow 0\right)$ because of the ac conductivity contribution already invoked in Sec. III A. At $600 \mathrm{~K}$, for example, one calculates $R_{\text {glass }}\left(=\rho_{\mathrm{dc} \text { glass }}\right)=980 \Omega \mathrm{m}$ and $f_{\max }=335 \mathrm{kHz}$, implying a conductivity $\sigma_{\mathrm{dc} \text { glass }} \approx 1$ $\times 10^{-3} \Omega^{-1} \mathrm{~m}^{-1}$ and a permittivity $\varepsilon_{\text {glass }} \approx 55$. At $630 \mathrm{~K}$, a shrinkness of the semicircle is observed, which may be interpreted by an increase of the conductivity of the material. As a matter of fact, the strong dual temperature-frequency dependence of the dc conductivity reported in Fig. 1(a) (Sec. III A) is mainly connected to the increase of the thermally activated conductivity, while the low frequency permittivity remains nearly constant $\left(\varepsilon_{\text {glass }} \approx 55\right)$. Its value, slightly 
higher than the one obtained from the $\varepsilon(\omega)$ spectrum at $1 \mathrm{MHz}(\approx 42 \pm 2)$, reflects only the high amount of the highly polarizable niobium ions in the glass.

Looking now at the poled glass Cole-Cole curves, one observes clearly two semicircles that indicate strong dielectric modifications induced by the poling treatment (Fig. 4). We have reported in a previous work that a $\mathrm{Na}^{+}$depleted surface layer appears at the anode side during the poling process. This additional NLO depleted layer, with thickness $\sim 3.5 \mu \mathrm{m}$, has been directly measured with a sodium content z-profile energy dispersive x-ray spectroscopy (EDS) study (see Fig. 4, lower part) and indirectly deduced from polarized SHG experiments. ${ }^{3}$ For a bulk plate containing one interfacial boundary between two layers, the equivalent electrical circuit may be considered as two parallel $R C$ elements connected in series. The corresponding Cole-Cole plot contains two arcs, as shown in Fig. 4 (see inset): the first arc (bulk layer) and the second arc $\left(\mathrm{Na}^{+}\right.$depleted layer) being associated with the $R_{1} C_{1}$ and $R_{2} C_{2}$ elements, respectively. Their relative position may be identified by their frequency range. Since the charge carriers responsible for the conductivity in sodium borophosphate glasses are mainly the sodium ions, the conductivity of the $\mathrm{Na}^{+}$poor layer is expected to be lower than that of the remaining bulk layer (i.e., $\sigma_{2}$ $\left.\ll \sigma_{1}\right)$. Although the NLO depleted layer is presumably very thin $\left(d_{2} / d_{1} \sim 1 / 200\right)$, one should expect $R_{2}>R_{1}$. Finally, since the relaxation time $\tau=1 / \omega_{\max }$ of the surface layer is the larger one, the arc due to the bulk layer lies on a frequency range higher than that of the thin NLO surface layer. This two-layer model that correctly describes our results at least qualitatively (dashed lines in Fig. 4) here is usually called the Maxwell-Wagner effect. ${ }^{17}$ Each layer (number 1 for the bulk and 2 for the sodium depleted region) is fully defined by three independent parameters: its conductivity $\sigma_{i}$, its dielectric permittivity $\varepsilon_{i}$, and its thickness $d_{i}$, which is known in the present case.

In order to get a more quantitative insight into this relaxation behavior, we have carried out a careful data analysis in the frequency space.

\section{Quantitative study of unpoled and poled glasses}

\section{Impedance measurements}

The imaginary part of the impedance $Z^{\prime \prime}$ of an unpoled and a poled plate are reported in Fig 5. For the unpoled sample, which evidences only one semicircle in the complex impedance plane (Fig. 4), one maximum is observed. As expected for the poled sample, two maxima are observed: a low frequency one, ascribed (as previously explained in Sec. III C) to the $\mathrm{Na}^{+}$poor and NLO active surface layer, and a high frequency one, related to the bulk layer. Applying the two-layer model (Maxwell-Wagner), $Z^{\prime \prime}$ is the sum of two terms:

$$
Z^{\prime \prime}=\sum_{i} \frac{C_{i} R_{i}^{2} \omega}{1+C_{i}^{2} R_{i}^{2} \omega^{2}}=\sum_{i} \frac{d_{i} / S}{\sigma_{i}^{2} /\left(\varepsilon_{0} \varepsilon_{i} \omega\right)+\varepsilon_{0} \varepsilon_{i} \omega},
$$

where $S$ is the electrode area, $\varepsilon_{0}$ is the vacuum permittivity, $i=1$ for the bulk $\left(d_{1}=730 \mu \mathrm{m}\right)$, and $i=2$ for the surface layer $\left(d_{2}=3.5 \mu \mathrm{m}\right)$.

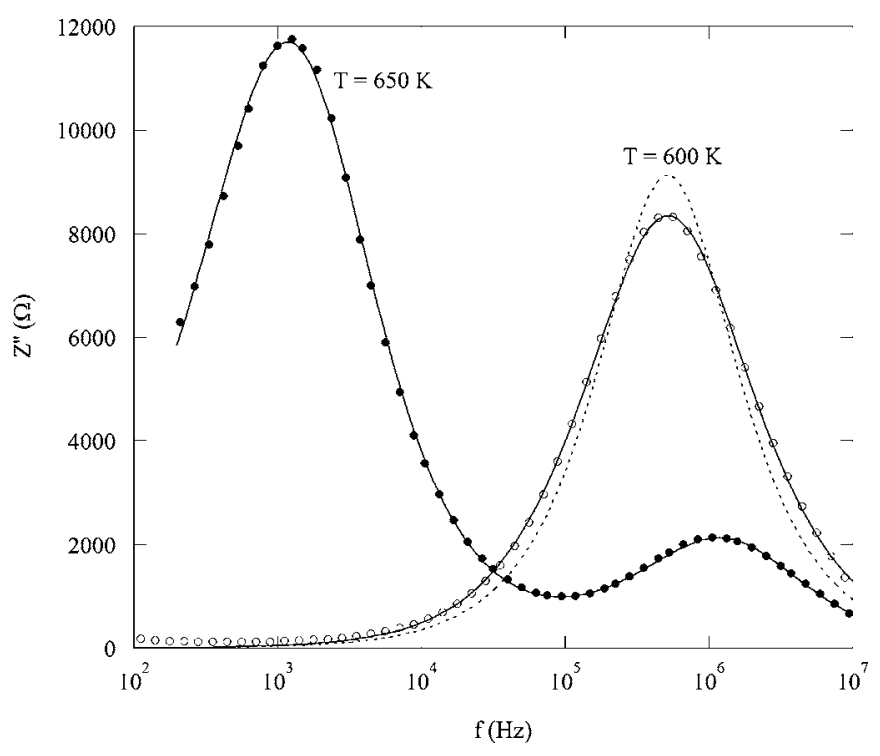

FIG. 5. Imaginary part of the impedance of an unpoled $(\bigcirc)$ plate at $600 \mathrm{~K}$ and of a poled plate $(0)$ at $650 \mathrm{~K}$. The best fits to the curves, obtained by neglecting (dashed line) or taking into account (solid line) the $A \omega^{n}$-conductivity term in Eq. (4), are also reported.

Let us consider first the unpoled glass. It may be modeled by one homogeneous layer, two parameters have thus to be fitted $\left(\sigma_{\mathrm{dc} \text { glass }}\right.$ and $\left.\varepsilon_{\text {glass }}\right)$ leading to $\sigma_{\mathrm{dc} \text { glass }}=1$ $\times 10^{-3} \Omega^{-1} \mathrm{~m}^{-1}$ and $\varepsilon_{\text {glass }}=54$ at $600 \mathrm{~K}$, in a close agreement with the values obtained from the $Z^{*}$ plot (Fig. 4), without the help of any mathematical treatment. However, the fit quality is poor (dashed line, Fig. 5). To give reasonable agreement with the experimental data, especially at high frequencies, we need to improve the model. Basically, the conductivity has already been considered frequency dependent, following Eq. (2) in Sec. III A. Although the introduction of a frequency dispersion of the conductivity increases the number of adjustable parameters up to four, the fitted curve (solid line, Fig. 5) is now satisfactory. The best parameters obtained at $600 \mathrm{~K} \quad\left(\sigma_{\mathrm{dc} \text { glass }}=1 \times 10^{-3} \Omega^{-1} \mathrm{~m}^{-1}, \quad A_{\text {glass }}=3\right.$ $\times 10^{-8}$ S.I., $n_{\text {glass }}=0.65$, and $\left.\varepsilon_{\text {glass }}=41\right)$ are in agreement with the $\sigma(\omega)$ analysis [Fig. 1(b)] and the direct high frequency measurement of $\varepsilon_{\text {glass }}$.

The same model improvement may then be applied to the poled sample and since it is a two-layer system, we have eight adjustable parameters to consider. Nevertheless, after a careful examination of the spectra recorded at different temperatures, it appears that some simplifications can be made without significantly changing the quality of the fit: the dispersion frequency $A_{2}$ and $n_{2}$ parameters may be neglected since the surface layer effect on the impedance occurs mainly at low frequencies. Thus, a reduced set of six parameters properly describes the imaginary part of the impedance $Z^{\prime \prime}$ of a poled glass, as shown in Fig. 5 .

\section{Conductivity measurements}

Let us now reconsider the conductivity $\sigma(\omega)$ of the poled sample reported for several temperatures in Fig. 6. As previously reported in Sec. III B, the $\sigma(\omega)$ frequency response of a poled glass is clearly different of that of an unpoled sample: a dielectric relaxation process is evidenced (see Fig. 


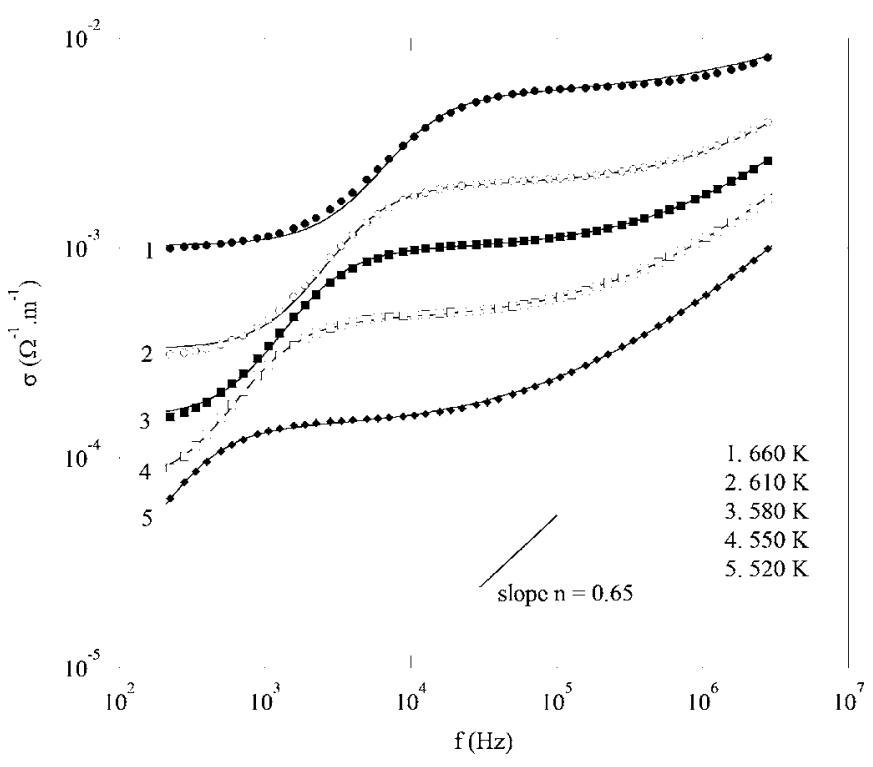

FIG. 6. Conductivity spectra $\sigma(\omega)$ obtained for a poled sodium borophosphate glass at different temperatures, together with the best fits (solid lines) deduced from Eq. (5).

2). Also, the frequency range of the relaxation process increases with temperature up to $650 \mathrm{~K}$, before thermal depoling occurs. In fact, for convenience and clarity, the whole data have been analyzed by fitting the $\sigma(\omega)$ spectra (Fig. 6) in the framework of the same model using the following formula (that can be deduced from the real part of the impedance $\left.Z^{\prime}\right)$ :

$$
\sigma(\omega)=\frac{\sigma_{0}+\sigma_{\infty} \omega^{2} \tau^{2}}{1+\omega^{2} \tau^{2}}+A \omega^{n}
$$

This equation is the sum of two terms: the first one ${ }^{18}$ describes the conductivity of a two-layer sample in which both the conductivity and the dielectric permittivity of each layer are frequency independent; the second term has been added to fit the data in the high frequency range $\left(>10^{4} \mathrm{~Hz}\right)$. The three parameters $\sigma_{0}$ [i.e., $\left.\sigma(\omega \rightarrow 0)\right], \sigma_{\infty}$, and $\tau$, the conductivity relaxation time, may be, in fact, expressed as a function of $\varepsilon_{1}, \varepsilon_{2}, \sigma_{1}$, and $\sigma_{2}{ }^{18}$ so that six parameters are still to be adjusted,

$$
\begin{aligned}
& \sigma_{\infty}=\frac{d_{1}+d_{2}}{\left(d_{1} / \varepsilon_{1}+d_{2} / \varepsilon_{2}\right)^{2}}\left(\frac{d_{1} \sigma_{1}}{\varepsilon_{1}^{2}}+\frac{d_{2} \sigma_{2}}{\varepsilon_{2}^{2}}\right), \\
& \sigma_{0}=\frac{d_{1}+d_{2}}{d_{1} / \sigma_{1}+d_{2} / \sigma_{2}}, \\
& \tau=\varepsilon_{0} \frac{\varepsilon_{1} d_{2}+\varepsilon_{2} d_{1}}{\sigma_{1} d_{2}+\sigma_{2} d_{1}} .
\end{aligned}
$$

The fitted curves are reported in Fig. 6 and the deduced parameters $\left[f=1 /(2 \pi \tau), \sigma_{1}\right.$ and $\left.\sigma_{2}\right]$ are reported as a function of $1 / T$ in Fig. 7, together with the conductivity of the unpoled glass. Concerning the permittivity $\varepsilon$, no appreciable change has been evidenced, neither between the two layers nor as a function of the temperature $\left(\varepsilon_{1} \approx \varepsilon_{2}=42 \pm 4\right)$. As expected, it is found that the conductivity of the bulk in the poled sample $\sigma_{1}$ is of the same order of magnitude as the

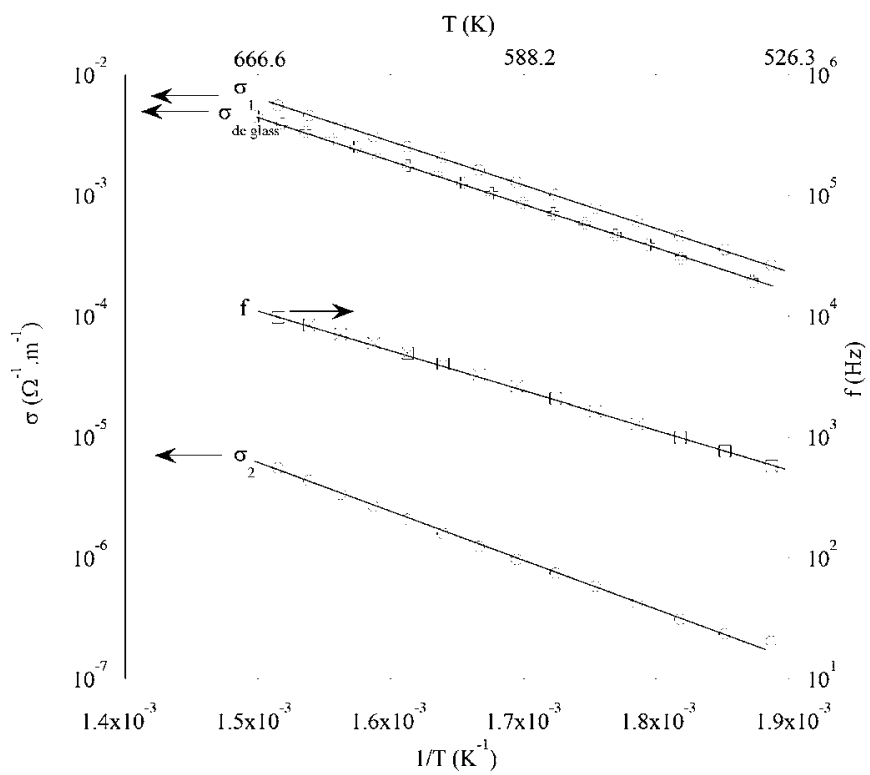

FIG. 7. Summary frequency plots of the fitted parameters from the $\sigma(\omega)$ curves of a poled sample: relaxation frequency of the conductivity and conductivities of the bulk $\sigma_{1}$ and of the surface layer $\sigma_{2}$. The dc conductivity of the unpoled glass is also reported for comparison purpose.

conductivity of the unpoled glass, $\sigma_{\mathrm{dc}}$ glass. In addition, the conductivity of the $\mathrm{Na}^{+}$depleted surface layer $\left(\sigma_{2}\right)$ is about $10^{3}$ times smaller than that of the bulk $\left(\sigma_{1}\right)$. It should also be noted that all these quantities are thermally activated and thus follow an Arrhenius law. In all the cases, the activation energy falls within the same range, namely, $0.72 \pm 0.06 \mathrm{eV}$. This is even true for $\sigma_{2}$, indicating that probably the same charge carrier (sodium ions) is responsible for the conduction in both the bulk and the surface layer; only their respective concentration would vary. This is also true for the relaxation frequency which, in fact, can be expressed ${ }^{18}$ as a linear combination of $\sigma_{1}$ and $\sigma_{2}$ [Eq. (6c)]. Similar activation energy values have been obtained in high sodium content borophosphate glasses. ${ }^{19}$

To summarize the $\sigma(\omega)$ analysis, a two-layer model for poled plates describes satisfactorily the experimental data in the whole frequency and temperature ranges investigated, giving realistic parameter values that describe the electrical properties of the $\mathrm{Na}^{+}$depleted surface layer. Such an investigation of a space charge effect on the dielectric properties of a poled glass has already been undertaken a few years ago, ${ }^{20,21}$ but for quite a different glass (silica). In this last study, the conductivity and the space charge formation was supposed to arise from unexpected impurities. ${ }^{8}$ However, in spite of some differences in the experimental methods, the same basic observations can be addressed: a space charge is induced by a thermal poling of glasses that become SHG active. The space charge can be reversibly eliminated, by simply annealing the sample in order to increase ionic diffusion: this thermal process induces the disappearance of both the dielectric relaxation and the nonlinear optical properties.

\section{CONCLUSION}

The dielectric investigations reported here have shown that poled glasses may be described as the sum of a bulk 
layer and a surface layer with lower $\mathrm{Na}^{+}$loading. The dielectric signature of unpoled and thermally depoled samples were identical, indicating that some reversible mechanisms occur upon heating. For both unpoled and poled samples, the dielectric permittivity of all layers remains unchanged $(\varepsilon \sim 42)$ in the $100 \mathrm{~Hz}-10 \mathrm{MHz}$ range. Although the conductivity of the $\mathrm{Na}^{+}$depleted surface layer in poled sample is three orders of magnitude lower than glass bulk layers (of poled and unpoled samples), the activation energy for all these layers ranges around $0.72 \mathrm{eV}$. Similar value of activation energy obtained from dc conductivity and impedance spectra suggests that the ion transport in the investigated glassy systems is due to hopping mechanism. Probably, the mobile charge carriers observed in our spectral range corresponds mainly to $\mathrm{Na}^{+}$. This is a clear example of poled glasses where an efficient NLO response is directly correlated to the conductivity of the material enhanced by a high alkali loading.

Yet, we still have to answer to the question of the charge compensation accompanying the depletion of sodium. Is there any anionic charge carrier (like oxygen) or a local charge redistribution, i.e., a structural distortion that compensates the $\mathrm{Na}^{+}$departure? An additional charge carrier would contribute to the conductivity of the material but not necessary to the dielectric constant in the presently frequency range investigated. Indeed, at the anode side under a high dc voltage, any anionic migration is improbable since the electric permittivity of these glasses is sufficiently high to induce an efficient electrical screening. On the other side, if any local charge redistribution occurs, it would not contribute to the conductivity of the glass but, although it could enhance locally the electric static polarizability of the medium, it is possible that it does not contribute to the macroscopic electric permittivity because of the collective nature of the glass response (cancellation effects).

${ }^{1}$ Y. Luo, A. Frauenglass, and S. R. J. Brueck, Appl. Phys. Lett. 84, 4935 (2004)

${ }^{2}$ M. Guignard, V. Nazabal, J. Troles, F. Smektala, H. Zeghlache, Y. Quiquempois, A. Kudlinski, and G. Martinelli, Opt. Express 13, 789 (2005).

${ }^{3}$ M. Dussauze, E. Fargin, M. Lahaye, V. Rodriguez, and F. Adamietz, Opt. Express 13, 4064 (2005).

${ }^{4}$ R. A. Myers, N. Mukherjee, and S. R. J. Brueck, Opt. Lett. 16, 1732 (1991).

${ }^{5}$ N. Mukherjee, R. A. Myers, and S. R. J. Brueck, J. Opt. Soc. Am. B 11, 665 (1994)

${ }^{6}$ T. G. Alley and S. R. J. Brueck, Opt. Lett. 23, 1170 (1998).

${ }^{7}$ O. Deparis, F. P. Mezzapesa, C. Corbari, P. G. Kazansky, and K. Sakaguchi, J. Non-Cryst. Solids 351, 2166 (2005).

${ }^{8}$ T. G. Alley, S. R. J. Brueck, and M. Wiedenbeck, J. Appl. Phys. 86, 6634 (1999).

${ }^{9}$ H. Imai, S. Horinouchi, N. Asakuma, K. Fukao, D. Matsuki, H. Hirashima, and K. Sasaki, J. Appl. Phys. 84, 5415 (1998).

${ }^{10}$ A. Narazaki, K. Tanaka, and K. Hirao, J. Opt. Soc. Am. B 19, 54 (2002).

${ }^{11}$ B. Ferreira, E. Fargin, J. P. Manaud, G. Le Flem, V. Rodriguez, and T. Buffeteau, J. Non-Cryst. Solids 343, 121 (2004).

${ }^{12}$ A. Malakho, M. Dussauze, E. Fargin, B. Lazoryak, V. Rodriguez, and F. Adamietz, J. Solid State Chem. 178, 1888 (2005).

${ }^{13}$ J. C. Dyre and T. B. Schrøder, Rev. Mod. Phys. 72, 873 (2000).

${ }^{14}$ D. L. Sidebottom, Phys. Rev. Lett. 83, 983 (1999).

${ }^{15}$ N. Godbout and S. Lacroix, J. Non-Cryst. Solids 316, 338 (2003)

${ }^{16}$ K. L. Ngai and S. W. Martin, Phys. Rev. B 40, 10550 (1989).

${ }^{17}$ R. Coelho, Physics of Dielectrics (Elsevier, New York, 1978).

${ }^{18}$ V. Daniel, Dielectric Relaxation (Academic, London, 1967).

${ }^{19}$ P. S. Anantha and K. Hariharan, Mater. Chem. Phys. 89, 428 (2005).

${ }^{20}$ M. Tomozawa and D.-W. Shin, J. Non-Cryst. Solids 241, 140 (1998).

${ }^{21}$ D.-W. Shin and M. Tomozawa, Phys. Chem. Glasses 42, 199 (2001). 